\title{
Parallel Edge Coloring of a Tree on a Mesh Connected Computer ${ }^{\star}$
}

\author{
Chang-Sung Jeong, Sung-Up Cho, Sun-Chul Whang, and Mi-Young Choi
}

Department of Electronics Engineering, Korea University

Anamdong 5-ka, Sungbuk-ku, Seoul 136-701, Korea

Tel: 82-2-3290-3229, Fax: 921-0544, E-mail: csjeong@charlie.korea.ac.kr

\begin{abstract}
An edge coloring is an assignment of colors to the edges of a graph so that no two edges with a common vertex have the same color. We show that, given an undirected tree $T$ with $n$ vertices, a minimum edge coloring of $T$ can be determined in $\mathrm{O}(\sqrt{n})$ time on a $\sqrt{n} \times \sqrt{n}$ meshconnected computer(MCC) by a novel technique which decomposes the tree into disjoint chains and then assigns the edge colors in each chain properly. The time complexity is optimal on MCC within constant factor.
\end{abstract}

\section{Introduction}

A mesh-connected computer(MCC) consists of $n$ identical processing elements( PE's) arranged on a $\sqrt{n} \times \sqrt{n}$ array, where each PE is connected to its four neighbors [1. (See Fig. 1.) We assume that MCC functions in SIMD mode, where all the PE's are synchronized under one control unit. MCC have been used as a model of parallel computation for problems in diverse areas including sorting, graph theoretic problems, computational geometry, image processing [2, 34567 . In this paper, we consider the problem of edge coloring in graph theory on MCC.

An edge coloring is an assignment of colors to the edges of a graph $G=(V, E)$ so that no two edges with a common vertex have the same color. A minimum edge coloring is an edge coloring which uses a minimum number of colors. Many researchers have worked on the design of sequential algorithms for finding a minimum edge coloring of a bipartite graph 10 11|12 1314. The best known sequential algorithm for edge coloring of a bipartite graph is due to Cole and Hopcroft [10] and runs in $\mathrm{O}(|E| \log |V|)$ time. For a minimum edge coloring of a tree $T$ with $n$ vertices, Mitchell and Hedetniemi presented an $\mathrm{O}(n)$ sequential algorithm [14].

Using the idea presented in 11,12, Lev, Pippenger and Valiant implemented an $\mathrm{O}\left(\log ^{3} n\right)$ parallel algorithm for a minimum edge coloring of a bipartite graph on EREW(Exclusive Read and Exclusive Write) computation model [13]. However, their algorithm cannot be directly implemented for tree on MCC in optimal time. As far as we know, no papers have proposed an optimal parallel

\footnotetext{
* This work has been supported by KOSEF and Brain Korea 21 projects
} 


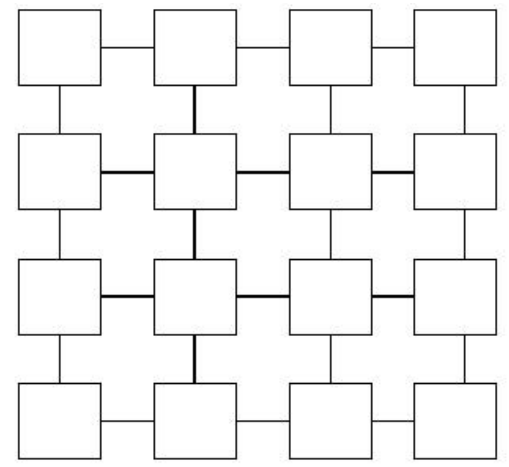

Fig. 1. $4 \times 4$ Mesh-connected computer

algorithm for the minimum edge coloring of a tree so far. In this paper, we consider the problem of minimum edge coloring of a tree with $n$ vertices and present an $\mathrm{O}(\sqrt{n})$ parallel algorithm for solving the problem on a $\sqrt{n} \times \sqrt{n}$ MCC, which is optimal within constant time factor. We shall show that this can be achieved by a novel technique which decomposes the tree into disjoint chains and then assigns the edge colors in each chain properly.

In section 2, we describe some notations and definitions, and in section 3 basic operations used in the design of the parallel algorithm. In section 4 , we give an $\mathrm{O}(\sqrt{n})$ parallel edge coloring algorithm for a tree $T(V, E),|V|=n$, on a $\sqrt{n} \times \sqrt{n}$ MCC and in section 5 we give a conclusion.

\section{Notations and Definitions}

Let $T(V, E)$ be a rooted directed tree, where there is a unique path from the root to each vertex in $V$. The vertices in $V$ are labeled from 1 to $|V|$. If $(i, j)$ is in edge set $E$, then $i$ is the parent of $j$, denoted by $p(j)$, and $j$ is a child of $i$. Note that for each vertex $i$, its parent $p(i)$ is unique, whereas it may have more than one child. The depth of a vertex $v$ in $V$ is the length(i.e., number of edges) of the path from the root to $v$. The degree of a vertex $v$ in $T$ is the number of the edges incident upon $v$ in $T$. Let $d$ be the maximum degree of a vertex in $T$. The minimum number of colors required in an edge coloring of a graph $G$ is called the edge chromatic number of $G$. It is well known that the edge chromatic number of a bipartite graph $G$ is equal to the maximum degree of $G$ [9]. Since a tree is a bipartite graph, the edge chromatic number of $T$ becomes $d$. Therefore, the minimum edge coloring of $T$ using colors 1 through $d$ partitions $E$ into $E_{1}, E_{2}, . ., E_{d}$ such that all the edges in $E_{i}$ are assigned color $i$ and no two edges in $E_{i}$ are adjacent to each other. (See Fig. 2,

The order number $s(i)$, for each edge $(p(i), i)$ in $T$, is defined as follows: We order each set $S$ of edges with the same parent by sorting the edges $(p(i), i)$ in $S$ in ascending order of $i$. For each edge $(p(i), i)$, we define $s(i)$ to be the number of 


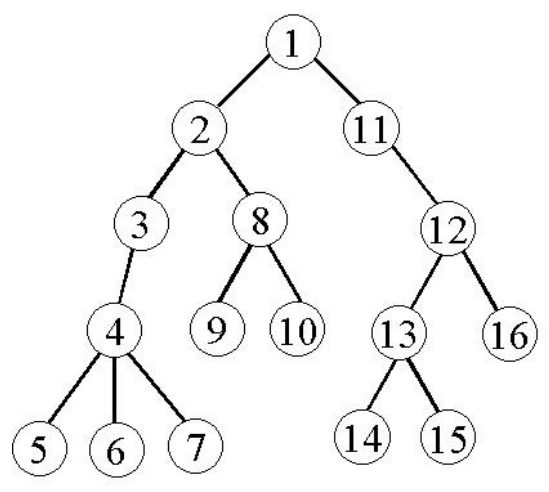

a) Tree

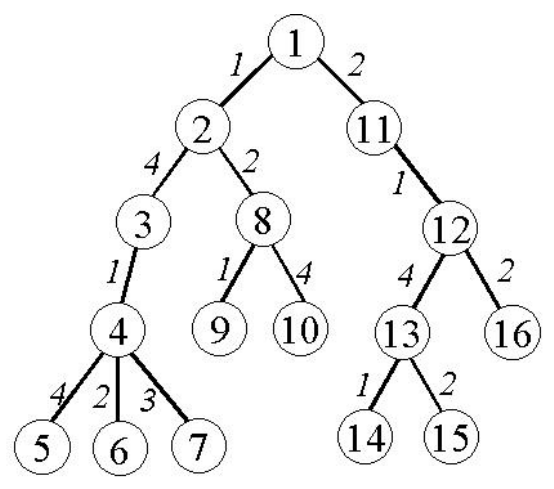

b) Minimum edge coloring

Fig. 2. Edge coloring of a tree with maximum degree 4

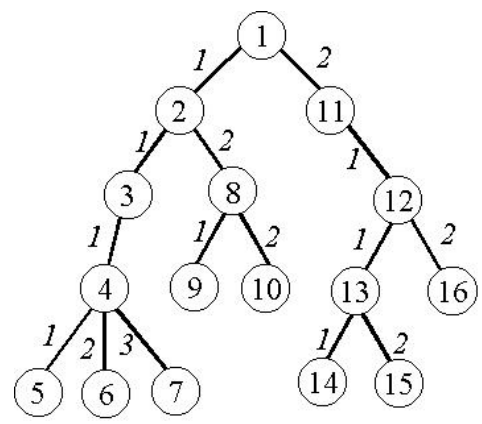

a) Order Numbers

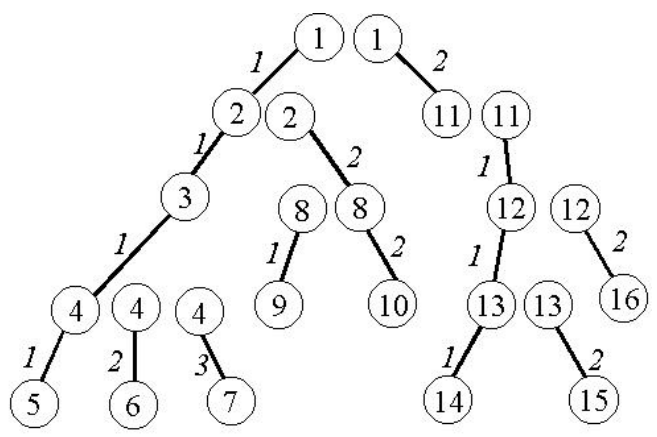

b) Color chains

Fig. 3. Order numbers and color chains

the edges in $S$ previous to it including itself. (See Fig. 3-a.) Suppose each edge in $T$ is colored with its order number. A path in $T$ is called a color chain if it is a maximal path consisting solely of edges with the same colors. (See Fig. 3-b.) A color chain consisting of edges with color $k$ is called $k$-chain.

A linear chain is a directed path which consists of edges each of the form $(i, \operatorname{succ}(i))$, where vertex $\operatorname{succ}(i)$ is the immediate successor of vertex $i$. (See Fig. 4) Suppose that each edge is associated with its weight. Then, the rank of an edge in the linear chain is the sum of the weights of its preceding edges and itself. Note that each color chain is a linear chain if $i$, for each edge $(p(i), i)$, is regarded as the immediate successor of $p(i)$. The root edge in the color chain(or linear chain) is the one with no predecessors. 


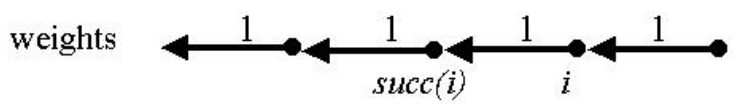

a) Linear chain

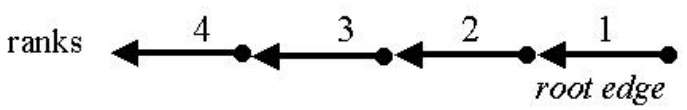

b) Ranks

Fig. 4. Rank in a linear chain

\section{Basic Operations}

MCC consists of $n$ PE's indexed from 0 to $n-1$, each having a local memory with a constant number of registers. We shall use the following basic operations which take $\mathrm{O}(\sqrt{n})$ time on a $\sqrt{n} \times \sqrt{n}$ MCC [23|5 8]. We assume that input data of size $n$ are distributed on a $\sqrt{n} \times \sqrt{n}$ MCC, one element per PE.

(1). Sorting: The elements are rearranged in nondecreasing or nonincreasing order of a specified key.

(2). Selected Broadcasting: Suppose a set $S$ of $n$ elements is partitioned into subsets $\left\{S_{1}, S_{2}, \ldots, S_{m}\right\}$. By selected broadcasting operation, the first element of each $S_{i}$ is distributed to all the other PE's containing the elements in $S_{i}$.

(3). RAR(Random Access Read): In a RAR, each PE specifies the key of data it wishes to receive, or it specifies a null key, in which case it receives nothing. Each PE reads a data from the PE which contains its specified key. Several PE's can request the same key and read the same data.

(4). RAW(Random Access Write): In a RAW, each PE specifies the key of data, and send its data to the PE which contains its specified key. If two or more PE's send data with the same specified key, then the PE with that key will receive the data with the minimium data or other value obtained by applying some commutative, associative binary operation to all the data sent to it.

(5). Chain-Rank: A linear chain consists of directed edges each of which is associated with its weight. The rank of each edge is defined as the sum of the weights of its predecessors in the chain. The first edge in the linear chain is called a root edge. Given a collection of linear chains with $n$ edges, chainrank operation finds, for each edge, its rank in the linear chain containing it. Figure 4 shows an example of linear chain and ranks of its edges each with weight 1. 


\section{Parallel Algorithm}

In this section we shall describe a parallel algorithm for finding a minimum edge coloring for a tree $T$. The basic idea of our algorithm can be described briefly as follows: First, color every edge of $T$ by its order number. Note that no more than two edges adjacent to a vertex in $T$ have the same color, and color $d$ have not been used at all, since the maximum value of order numbers is $d-1$. Using those facts, we can obtain a minimum edge coloring by decomposing $T$ into disjoint color chains and then changing the colors of every other edge in each color chain into $d$. In the following we shall describe the detailed implementation of the parallel algorithm.

\section{Parallel Algorithm Edge-Coloring(EC)}

Input: Each PE with index $i$ on a $\sqrt{n} \times \sqrt{n}$ MCC contains an edge $(p(i), i)$ and a vertex $i$ of a directed tree $T(V, E)$.

Output: A minimum edge coloring $C$ such that $C(p(i), i)$ is $m$ if the edge $(p(i), i)$ is assigned color $m$.

1): Find a maximum degree $d$ for $T$. This can be done as follows: Send, for each PE containing an edge $(p(i), i), 1$ to the PE containing a vertex $p(i)$ by RAW. During RAW, several 1's may have the same destination PE, and the $\mathrm{PE}$ containing a vertex $j$ can receive the value obtained by summing all the 1's sent to that PE. Therefore, each PE containing a vertex $j$ stores the number of its children after executing RAW, and hence we can compute the degree of $j$ by adding one to it. Then, we can find easily the maximum degree $d$ by computing the largest value of degrees of all the vertices.

2): Compute, for each edge $(p(i), i)$, its order number $s(i)$.

Implementation: First, sort every edge $(p(i), i)$ lexicographycally according to $(p(i), i)$ in nondecreasing order. During sort the smallest edge $e$ in each set $S$ of edges with the same parent is located and index $I$ of PE containing $e$ is sent to all the PE's storing the other edges in $S$ by selected broadcasting. Then, $s(i)$ can be obtained by subtracting $(I-1)$ from the PE index containing $(p(i), i)$.

3): Assign, for each edge $(p(i), i)$, color $s(i)$ to $C(p(i), i)$. (See Fig. [3-a.) The colors assigned at this step range from 1 to $d-1$, since the maximum value of $s(i)$ is $d-1$.

4): Decompose $T$ into disjoint k-chains, $1 \leq k \leq d-1$. (See Fig. 3 -b.)

Implementation: Step 4) can be done by seperating the root edge in each color chain from the other color chains. This can be implemented as follows: Find, for each edge $(p(i), i)$, its parent edge $(j, p(i))$ by RAR, where $j$ is a parent of $p(i)$. Then, check whether the color of $(p(i), i)$ is different from that of $(j, p(i))$ or not. If it is, seperate $(p(i), i)$ from the other color chains adjacent to it by replacing $(p(i), i)$ with $\left(p(i)_{s(i)}, i\right)$, since $(p(i), i)$ becomes a root edge of the color chain containing it; otherwise $(p(i), i)$ cannnot be a root edge of 


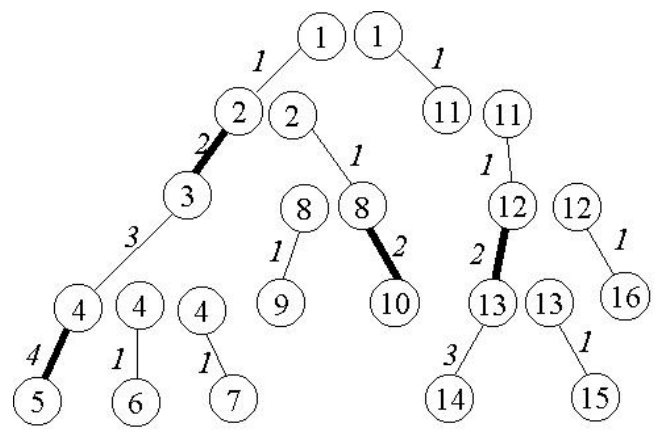

a) Ranks in color chain : Bold lines represent edges with even ranks

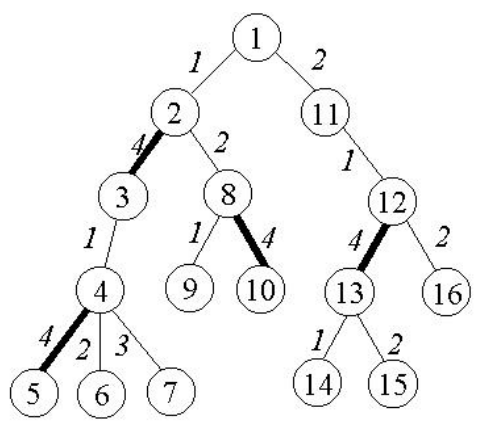

b) Minimum edge coloring

Fig. 5. Example of the parallel algorithm EC

the color chain to which it belongs, and hence we don't have to separate it from the other color chains.

5): Find, for each edge $(p(i), i)$, its rank in the color chain containing it after assigning a weight 1 to every edge. (See Fig. 55.)

Comment: We can easily see that at most two edges with a common vertex may have the same color after executing step 3). In order to fix the conflicting color assignments, the colors of all the edges with even ranks are replaced with color $d$ in the following step.

6): Change, for each edge $(p(i), i)$ with the even rank, its color $C(p(i), i)$ into $d$. (See Fig. 5tb.)

\section{End Algorithm.}

Lemma 1: The parallel algorithm EC can be executed in $\mathrm{O}(\sqrt{n})$ time on a $\sqrt{n} \times \sqrt{n} \mathrm{MCC}$.

Proof: Step 1) and 2) take $\mathrm{O}(\sqrt{n})$ time for RAW, sorting, and selected broadcasting. Step 3) can be done in $\mathrm{O}(1)$ time and step 4) in $\mathrm{O}(\sqrt{n})$ time for RAR. Step 5) can be carried out in $\mathrm{O}(\sqrt{n})$ time by chain-rank operation. Step 6) takes $\mathrm{O}(1)$ time. Therefore, the overall time complexity is $\mathrm{O}(\sqrt{n})$ time.

Lemma 2: The edge coloring $C$ in the parallel algorithm EC is a minimum edge coloring for $T$.

Proof: Clearly, the colors used in the parallel algorithm EC range from 1 to $d$. Since $d$ is a edge chromatic number of $T$, all we have to prove is that no two edges with a common vertex have the same color. Consider a vertex $i$ with parent $p(i)$ and $m$ children $j_{1}, j_{2}, j_{3}, . ., j_{m}$ ordered from left to right so that the order number of $\left(i, j_{k}\right)$ is $k, 1 \leq k \leq m$. (See Fig. 6 6.) Let $A$ be a set of edges $\left(i, j_{k}\right), 1 \leq k \leq m$. After step 3$)$ each edge $\left(i, j_{k}\right)$ is assigned color $k$ in the range 


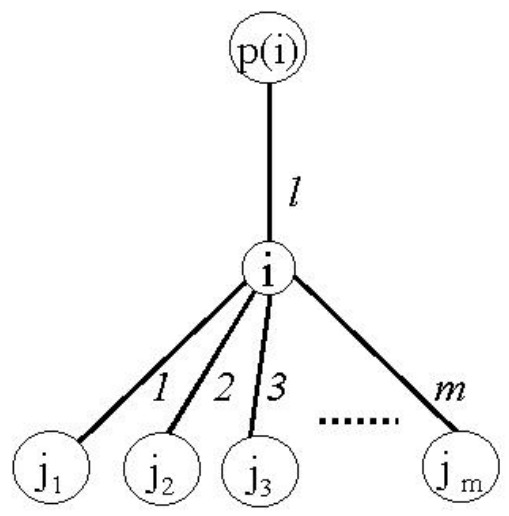

a)

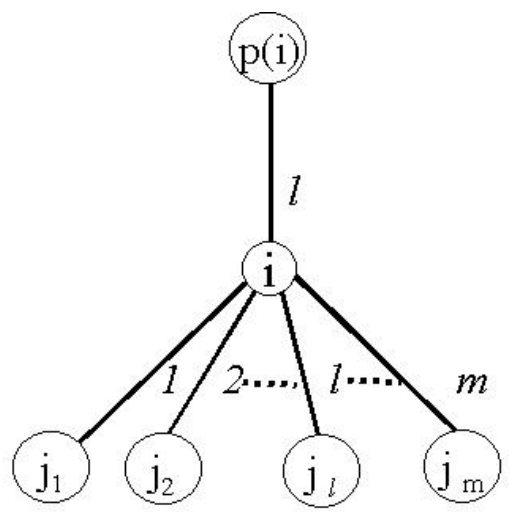

b)

Fig. 6. Illustration of Lemma 2

from 1 to $d-1$. Suppose that edge $(p(i), i)$ is colored $\ell$. Then, we can cosider the following two cases:

Case 1): $\ell>m$ : Since all the edges in $A$ are assigned colors all different from $\ell$, each edge in $A$ becomes a root edge in the color chain containing it and has its rank value 1 . Therefore, the color of each edge in $A$ is not changed at step 6 ), and hence no two edges adjacent to $i$ have the same color.

Case 2): $\ell \leq m$ : Let $\left(i, j_{\ell}\right)$ denote an edge with the same color $\ell$ as $(p(i), i)$. (See Fig. 6rb.) Since both of $(p(i), i)$ and $\left(i, j_{\ell}\right)$ belong to the same $\ell$-chain, one of them changes its color $\ell$ to $d$ at step 6$)$. All the edges in $A$ other than $\left(i, j_{\ell}\right)$ have different colors from $\ell$, and their colors are not changed due to the fact that each of them is a root edge in the color chain containing it. Therefore, no two edges adjacent to $i$ have the same color.

An undirected tree with $n$ vertices can be converted into the directed one in $\mathrm{O}(\sqrt{n})$ time on a $\sqrt{n} \times \sqrt{n}$ MCC [5]. Therefore, the following theorem follows directly from lemma 1 and 2 .

Theorem 1: Given an undirected tree $T$ with $n$ vertices, its minimum edge coloring can be computed in $\mathrm{O}(\sqrt{n})$ time on a $\sqrt{n} \times \sqrt{n}$ MCC.

\section{Conclusion}

In this paper, we have presented the parallel algorithm for finding the minimum edge coloring of a tree with $n$ vertices in $\mathrm{O}(\sqrt{n})$ time on a $\sqrt{n} \times \sqrt{n}$ MCC. The time complexity is optimal on MCC within constant factor. This was achieved by the smart technique which decomposes the tree into disjoint color chains and then changes the colors of each color chain properly. It remains open if one 
can find an $\mathrm{O}(\sqrt{n})$ algorithm for finding a maximum matching of a tree with $n$

vertices on a $\sqrt{n} \times \sqrt{n}$ MCC. Also open are the problems for finding a minimum edge coloring and maximal matching for a general bipartite graph on a $\sqrt{n} \times \sqrt{n}$ MCC in $\mathrm{O}(\sqrt{n})$ time. We expect that our parallel algorithm developed in this paper may provide a base for finding the optimal parallel algorithms for those open problems.

\section{References}

1. H. J. Siegel, A Model of SIMD Machines and a Comparison of Various Interconnection Networks, IEEE Trans. Comput. 28 (1979) 907-917.

2. C. S. Jeong, and D. T. Lee, Parallel Geometric Algorithms on a Mesh-Connected Computer, Algorithmica 5 (1990) 155-177.

3. C. D. Thompson, and H. T. Kung, Sorting on a Mesh-Connected Parallel Computer, Comm. ACM 20 (1977) 263-271.

4. N. Folwell, S. Guha and I. Suzuki, A Practical Algorithm for Integer Sorting on a Mesh-Connected Computer, Parallel Algorithms and Applicationsi 12 (1997) 265278.

5. M. M. Atallah, and S. E. Hambrusch, Solving Tree Problems on a Mesh-Connected Processor Array, Proc. 26th IEEE FOCS Conference (1985) 222-231.

6. M. H. Kim, and C. S. Jeong, An Optimal Parallel Matching Algorithm for a Convex Bipartite Graph on a Mesh-Connected Computer, Parallel Algorithms and Applications 5 (1995) 15-35.

7. B. Murthy, K. Bhuvaneswari, and C. S. Murthy, A New Algorithm based on Givens Rotations Solving Linear Equations on Fault-Tolerant Mesh-Connected Processors, IEEE Trans. on Parallel and Distributed Systems 9 (1998) 825-832.

8. D. Nassimi, and S. Sahni, Data Broadcasting in SIMD Computers, IEEE Trans. on Comput. (1981) 101-106.

9. J. A. Bondy, and U. S. R. Murty, Graph Theory with Applications, American Elsevier (1977).

10. R. Cole, and J. Hopcroft, On Edge Coloring Bipartite Graphs, SIAM J. Comput. 11 (1982) 540-546.

11. H. Gabow, Using Euler Partitions to Edge Color Bipartite Multigraphs, Inter. J. Comp. and Inf. Scie. 5 (1976) 345-355.

12. H. Gabow, and O. Kariv, Algorithms for Edge Coloring Bipartite Multigraphs, SIAM J. Comput. 11 (1982) 117-129.

13. G. Lev, N. Pippenger, and L. G. Valiant, A Fast Parallel Algorithm for Routing in Permutation Networks, IEEE Trans. Comput. 30 (1981) 93-110.

14. S. Mitchell, and S. Hedetniemi, Linear Algorithms for Edge coloring Trees and Unicyclic Graphs, Info. Proc. Lett 9 (1979) 110-112. 